\title{
Trajectory Following Method on Output Regulation of Affine Nonlinear Control Systems with Relative Degree not Well Defined
}

\author{
Janson Naiborhu \\ Industrial Financial Mathematics Group \\ Faculty of Mathematics and Natural Science, ITB. \\ Email: janson@math.itb.ac.id
}

\begin{abstract}
The problem of output regulation of affine nonlinear systems with the relative degree not well defined by modified steepest descent control is studied. The modified steepest descent control is a dynamic feedback control which is generated by the trajectory following method. By assuming the system is minimum phase, output of the system can be regulated globally asymptotically.
\end{abstract}

Keywords: minimum phase; modified steepest descent control; trajectory following method.

\section{$1 \quad$ Introduction}

This paper is concerned with the problem of output feedback regulation of nonlinear systems. Many authors have considered such problems, using various approaches. Some necessary and sufficient conditions for regulation via static output feedback are established in [1], by extending previous results of Artstein [2] and Sontag [3]. For a class of nonlinear systems, Pomet et al. [4] developed sufficient conditions for the global stabilization of the observed state via dynamic output feedback by using a Lyapunov-based technique.

Vincent and Grantham [5] studied two Lyapunov optimizing approaches for designing feedback controllers. One of them is the steepest descent control. The steepest descent control corresponds to specifying a preferred direction of motion, opposite to the gradient of a specified scalar-valued "descent function". The steepest descent control is effective at yielding global asymptotic stability [6]. The related research is found in [7-9] which has proposed the direct gradient descent control.

Based on Grantham's idea [5-6] we study the output regulation of affine nonlinear systems (SISO) by using modified steepest descent control. First we define the descent function as a squared form. Then we apply the trajectory following method [5] to get modified steepest descent control which forms a dynamic feedback controller. This control is different with controller in [7-9]. In

Received August $30^{\text {th }}, 2010$, Revised October $19^{\text {th }}, 2010,2^{\text {nd }}$ Revision November $19^{\text {th }}, 2010$, Accepted for publication December $21^{\text {st }}, 2010$. 
[7], Naiborhu et al., developed the direct gradient descent control (dgdc) for stabilization of nonlinear systems through gateaux differential and in [8-9], Shimizu et al., improve the dgdc for tracking output of nonlinear system with relative degree well defined by added a virtual control in dgdc.

By direct application of necessary condition for a minimum, we get the static control law which is the same as the control law obtained by input-output linearization technique [10]. This static control law is effective at yielding local asymptotic stability. In this paper however, we show that the modified steepest descent control regulates the output of the system globally asymptotically.

\section{Problem Statement and Assumptions}

Consider SISO affine nonlinear control system

$$
\begin{aligned}
& \dot{x}=f(x)+g(x) u, \\
& y=h(x)
\end{aligned}
$$

where $x \in R^{n}$ is the state vector, $u \in R$ is the control input and $y \in R$ is the measured output. $f: R^{n} \rightarrow R^{n}$ is a smooth function with $f(0)=0$, $g: R^{n} \rightarrow R^{n}$ and $h: R^{n} \rightarrow R$ are smooth functions. Assume also that $h(0)=0$.

Our objective is to make the output $y(t)$ go to zero while keeping the state bounded. The main task is to design control $u$ such that $y(t) \rightarrow 0$ as $t \rightarrow \infty$. For keeping the state bounded we assume that system (1) is minimum phase, i.e. if a nonlinear state feedback can hold the output of the system identically zero and the internal dynamics of the system is asymptotically stable [11].

For designing control input $u$ we need an explicit relationship between the output and the input. This relationship is defined as relative degree of the system. In the following we write the definition of relative degree of the system by Isidori [10]

Definition 1 The nonlinear system (1)-(2) is said to have a relative degree $r$ at a point $x^{a}$ if $\frac{\partial y^{(k)}}{\partial u}=0$ for all $x$ in a neighborhood of $x^{a}$ and all $k \leq r-1$, and $\frac{\partial y^{(r)}}{\partial u} \neq 0$.

Definition 1 means that we need to differentiate the output of a system $r$ times to generate an explicit relationship between the output $y$ and input $u$. Let the 
relative degree of the system be $r$. Then $y, \dot{y}, \cdots, y^{(r-1)}$ are functions of $x, y^{(r)}$ is a function of $x$ and $u$ and

$$
\begin{aligned}
& \frac{\partial h(x)}{\partial x} g(x)=0, \frac{\partial \beta^{1}(x)}{\partial x} g(x)=0, \cdots, \\
& \frac{\partial \beta^{r-2}(x)}{\partial x} g(x)=0, \frac{\partial \beta^{r-1}(x)}{\partial x} g(x) \neq 0,
\end{aligned}
$$

where $\beta^{j}(x)=y^{(j)}, j=1, \cdots, r-1$. Furthermore, we have

$$
y^{(r)}=\frac{\partial \beta^{r-1}(x)}{\partial x} f(x)+\frac{\partial \beta^{r-1}(x)}{\partial x} g(x) u .
$$

Consider equation (3). If $\frac{\partial \beta^{r-1}\left(x^{s}\right)}{\partial x} g\left(x^{s}\right)=0$, then the relative degree of the system is not $r$ at $x^{s}$. In other words, input $u$ can not influence the output $y$. Thus, we can not do anything to the control input $u$ so that $y$ becomes zero.

Let us assume that the relative degree of the system (1)-(2) is $r$. Then we have Eq. (3) and $\frac{\partial \beta^{r-1}(x)}{\partial x} g(x) \neq 0$. From input-output linearization technique [10], control input $u$ is chosen as

$$
u=-\frac{c_{r} \frac{\partial \beta^{r-1}(x)}{\partial x} f(x)+\left(\sum_{j=0}^{r-1} c_{j} y^{(j)}\right)}{c_{r} \frac{\partial \beta^{r-1}(x)}{\partial x} g(x)}
$$

if $\frac{\partial \beta^{r-1}(x)}{\partial x} g(x) \neq 0$

A problem occures if $\frac{\partial \beta^{r-1}(x)}{\partial x} g(x)=0$ at a point $x^{s}$ and the static control law (4) can not be applied. To avoid this difficulty, many researchers assume that system has relative degree $r$ well defined, i.e. for all $x \in R^{n}$ relative degree of the system is $r$. But if this assumption can not be satisfied, Hirschorn and Davis [12] introduced the degree of singularity to solve this problem.

In [10], Isidori shows that by assuming the zero dynamics of the system be locally asymptotically stable we can choose a static control law such that output of the system goes to zero while keeping the state of the system bounded 
locally. In this paper we want to extend this result globally by using the modified steepest descent control. The modified steepest descent control is a dynamic feedback control of the form

$$
\dot{u}=-\frac{d F}{d u}+v,
$$

where $F$ is a descent function and $v$ is an artificial input ( $F$ will be defined in section 3 and $v$ will be determined such that the descent function $F$ decreases).

Thus, in this paper we show that by assuming

Assumption 1 System (1) is minimum phase, the modified steepest descent control (5) will render the output of the system (1)-(2) to zero if time $t$ goes to infinity even if the relative degree of the system not well defined.

\section{$3 \quad$ Trajectory Following Method}

The trajectory following method [5] is a numerical optimization method based on solving continuous differential equations. This method is "trajectory following" in the sense that we move from an initial guess for the solution to a solution point along a trajectory generated by a set of differential equations.

Consider first the problem of minimizing performance function $G(u)$, $G: R^{r} \rightarrow R$, subject to no constraints. Let $u^{*}$ provide a local minimum for performance function $G(u)$ and use the local minimum point $u^{*}$ as an initial condition for integrating the differential equation,

$$
\dot{u}=f(u)
$$

where $f(\cdot)$ is a function at our disposal, to be determined shortly. Calculate the time derivative of $G(u)$ along the trajectory generated by the solution to (6).

Then at $u=u^{*}$

$$
\left.\frac{d G}{d t}\right|_{u^{*}}=\left.\frac{\partial G}{\partial u}\right|_{u^{*}} f\left(u^{*}\right) \geq 0 .
$$

Since we are interested in a trajectory that will search for a minimum, the above observation suggests that we integrate (6) by choosing

$$
f(u)=-\left[\frac{\partial G}{\partial u}\right]^{T}
$$


and equation (7) becomes

$$
\frac{d G}{d t}=\frac{\partial G}{\partial u} f(u)<0, \forall u \neq u^{*}
$$

Thus, in order to solve the problem of minimizing $G(\cdot)$ in an unconstrained control space, one need only choose an appropriate initial condition for $u$ and integrate

$$
\dot{u}=-\left[\frac{\partial G}{\partial u}\right]^{T}
$$

until the equilibrium solution is achieved.

\section{$4 \quad$ Modified Steepest Descent Control}

We design control law $u$ through properties of the solution of higher order ordinary differential equation. Consider a differential equation

$$
c_{r} y^{(r)}(t)+c_{r-1} y^{(r-1)}(t)+\cdots+c_{1} \dot{y}(t)+c_{0} y(t)=0
$$

where $r$ is the relative degree of the system (1)-(2). If a polynomial

$$
p(s)=c_{r} s^{r}+c_{r-1} s^{r-1}+\cdots+c_{1} s+c_{0}
$$

is Hurwitz, then a solution of differential equation (11) tends to zero if $t \rightarrow \infty$.

Remark 1 Constants $c_{i}$ can be chosen such that the polynomial (12) is Hurwitz, i.e. the polynomial has all roots strictly in the left-half plane. So we have freedom to choose the rate of convergence that the output goes to zero.

Define a descent function

$$
F\left(y(t), \dot{y}(t), \cdots, y^{(r)}(\mathrm{t})\right)=\left(\sum_{j=0}^{r} c_{j} y^{(j)}(t)\right)^{2}
$$

where $y(t), \dot{y}(t), \cdots, y^{(r)}(t)$ are bounded. Reasons why we define the descent function as in (13) are :

1. The control input $u$ can be designed if there exists an explicit relationship between input $u(t)$ and output $y(t)$. Thus, descent function (13) must be a function of $y^{(r)}(t)$. 
2. We need that the descent function $F\left(y(t), \dot{y}(t), \cdots, y^{(r)}(t)\right)$ is a quadratic form and that $F(0,0, \cdots, 0)$ must equal zero. Accordingly, the minimum value of descent function $F\left(y(t), \dot{y}(t), \cdots, y^{(r)}(t)\right)$ is zero. If $F\left(y(t), \dot{y}(t), \cdots, y^{(r)}(t)\right)$ is zero, then $\sum_{j=0}^{r} c_{j} y^{(j)}(t)=0$. Furthermore, if polynomial (12) is Hurwitz, then $y$ tends to zero.

Our objective is to find a control law $u(t)$ for all $t$ such that the descent function $F\left(y(t), \dot{y}(t), \cdots, y^{(r)}(t)\right)$ becomes minimum and state $x(t)$ is at least bounded. Then our problem is formulated as follows.

$$
\begin{gathered}
\min _{u(t)} F\left(y(t), \dot{y}(t), \cdots, y^{(r)}(t)\right) \\
\text { subj.to } \dot{x}(t)=f(x(t))+g(x(t)) u(t) \\
y(t)=h(x(t)) .
\end{gathered}
$$

When $u$ is a scalar, a necessary condition for a local minimum is given as follows.

$$
\frac{d F\left(y, \dot{y}, \cdots, y^{(r)}\right)}{d u}=0,
$$

where

$$
\frac{d F\left(y, \dot{y}, \cdots, y^{(r)}\right)}{d u}=2 c_{r}\left(\sum_{j=0}^{r} c_{j} y^{(j)}\right) \frac{\partial \beta^{r-1}(x)}{\partial x} g(x) .
$$

From the necessary condition (17) we have

$$
\sum_{j=0}^{r} c_{j} y^{(j)}=0
$$

or

$$
\frac{\partial \beta^{r-1}(x)}{\partial x} g(x)=0
$$

By direct application of the necessary condition (19) for a minimum we get the control law (4).

In the following we use a numerical technique to solve the minimization problem(14)-(16) at every point of a trajectory. One numerical techniques is the 
trajectory following method which have been reviewed in section 3. The idea of the trajectory following method is to solve the numerical optimization problem only once, at the initial point of the trajectory. For all other points $x$ along a trajectory, the control $u$ is determined from the differential equation

$$
\begin{aligned}
\dot{u} & =-\frac{d F}{d u} \\
& =-2 c_{r}\left(\sum_{j=0}^{r} c_{j} y^{(j)}\right) \frac{\partial \beta^{r-1}(x)}{\partial x} g(x) .
\end{aligned}
$$

The control law in equation (21) is called the steepest descent control.

Calculate the time derivative of descent function (13) along the trajectory of the extended system

$$
\begin{aligned}
\dot{x} & =f(x)+g(x) u, \\
\dot{u} & =-2 c_{r}\left(\sum_{j=0}^{r} c_{j} y^{(j)}\right) \frac{\partial \beta^{r-1}(x)}{\partial x} g(x) .
\end{aligned}
$$

Then we have

$$
\begin{aligned}
& \dot{F}\left(y, \dot{y}, \cdots, y^{(r)}\right) \\
& \quad=2\left(\sum_{j=0}^{r} c_{j} y^{(j)}\right)\left(\sum_{j=1}^{r} c_{j-1} y^{(j)}+c_{r} \frac{d}{d t}\left(y^{(r)}\right)\right) .
\end{aligned}
$$

By substituting (3) and (21) into (24), we have

$$
\begin{aligned}
& \dot{F}\left(y, \dot{y}, \cdots, y^{(r)}\right) \\
& =2\left(\sum_{j=0}^{r} c_{j} y^{(j)}\right)\left[\sum_{j=1}^{r} c_{j-1} y^{(j)}+c_{r}\left\{\frac{d}{d t}\left(\frac{\partial \beta^{r-1}(x)}{\partial x} f(x)\right)\right.\right. \\
& \left.\left.+\frac{d}{d t}\left(\frac{\partial \beta^{r-1}(x)}{\partial x} g(x)\right) u\right\}\right]-4 c_{r}^{2}\left(\sum_{j=0}^{r} c_{j} y^{(j)}\right)^{2}\left(\frac{\partial \beta^{r-1}(x)}{\partial x} g(x)\right)^{2} .
\end{aligned}
$$

From equation (25), the value of time derivative of descent function (13) along the trajectory of (22)-(23) can not be guaranteed to be less than zero for $t \geq 0$. Consider the extended system (22)-(23) and time derivative of descent function (25). We do not have a variable which will be used to push the time derivative of descent function (25) less than zero. 
Now we modify the steepest descent control (23) by adding an artificial input $v$. Then the extended system (22)-(23) becomes

$$
\begin{aligned}
& \dot{x}=f(x)+g(x) u, \\
& \dot{u}=-2 c_{r}\left(\sum_{j=0}^{r} c_{j} y^{(j)}\right) \frac{\partial \beta^{r-1}(x)}{\partial x} g(x)+v .
\end{aligned}
$$

The control law in equation (27) is called as modified steepest descent control. The similar method using artificial input can be seen in [8].

By the same way, let us calculate time derivative of descent function (13) along the trajectory of (26)-(27) yielding

$$
\begin{aligned}
& \dot{F}\left(y, \dot{y}, \cdots, y^{(r)}\right) \\
& =2\left(\sum_{j=0}^{r} c_{j} y^{(j)}\right)\left[\sum_{j=1}^{r} c_{j-1} y^{(j)}+c_{r}\left\{\frac{d}{d t}\left(\frac{\partial \beta^{r-1}(x)}{\partial x}\right)\right.\right. \\
& \left.\left.+\frac{d}{d t}\left(\frac{\partial \beta^{r-1}(x)}{\partial x} g(x)\right) u\right\}\right]-4 c_{r}^{2}\left(\sum_{j=0}^{r} c_{j} y^{(j)}\right)^{2}\left(\frac{\partial \beta^{r-1}(x)}{\partial x} g(x)\right)^{2} \\
& +2 c_{r}\left(\sum_{j=0}^{r} c_{j} y^{(j)}\right)\left(\frac{\partial \beta^{r-1}(x)}{\partial x} g(x)\right) v .
\end{aligned}
$$

Consider equation (28). We can choose the artificial input $v$ such that $\dot{F}\left(y, \dot{y}, \cdots, y^{(r)}\right)$ be less than zero. If we take

$$
v=\left\{\begin{array}{lll}
k\left(x, y, \cdots, y^{r}, u\right) & \text { if } & \frac{\partial \beta^{r-1}(x)}{\partial x} g(x) \neq 0 \\
0 & \text { if } & \frac{\partial \beta^{r-1}(x)}{\partial x} g(x)=0
\end{array}\right.
$$

where

$$
\begin{aligned}
& k\left(x, y, \cdots, y^{r}, u\right) \\
& \quad=-\left[c_{r}\left(\frac{\partial \beta^{r-1}(x)}{\partial x} g(x)\right)\right]^{-1}\left[\sum_{j=1}^{r} c_{j-1} y^{(j)}+c_{r}\left\{\frac{d}{d t}\left(\frac{\partial \beta^{r-1}(x)}{\partial x} f(x)\right)\right.\right.
\end{aligned}
$$




$$
\left.\left.+\frac{d}{d t}\left(\frac{\partial \beta^{r-1}(x)}{\partial x} g(x)\right) u\right\}\right]
$$

then

$$
\begin{aligned}
& \dot{F}\left(y, \dot{y}, \cdots, y^{(r)}\right) \\
& \quad=-4 c_{r}^{2}\left(\sum_{j=0}^{r} c_{j} y^{(j)}\right)^{2}\left(\frac{\partial \beta^{r-1}(x)}{\partial x} g(x)\right)^{2}
\end{aligned}
$$

if $\frac{\partial \beta^{r-1}(x)}{\partial x} g(x) \neq 0$ and less than zero for nonzero $\sum_{j=0}^{r} c_{j} y^{(j)}$.

Consider the descent function (13) and its time derivative (31). The descent function (13) is bounded because $y, \dot{y}, \cdots, y^{(r)}$ are bounded. If we take $v$ as in (29) then the descent function (13) will decrease until $\sum_{j=0}^{r} c_{j} y^{(j)}$ becomes zero. Adding artificial input $v$ into dynamic controller (23) is used to guarantee the descent function (13) decrease to zero $\left(\sum_{j=0}^{r} c_{j} y^{(j)}=0\right)$. By this reason, it is realistic to put the value of $v$ becomes zero if $\sum_{j=0}^{r} c_{j} y^{(j)}=0$. Thus, the equation (29) becomes

$$
v=\left\{\begin{array}{lll}
k\left(x, y, \cdots, y^{r}, u\right) & \text { if } & \frac{\partial \beta^{r-1}(x)}{\partial x} g(x) \neq 0 \\
& & \text { or } \sum_{j=0}^{r} c_{j} y^{(j)} \neq 0 \\
0 & \text { if } \quad & \frac{\partial \beta^{r-1}(x)}{\partial x} g(x)=0 \\
& & \text { or } \sum_{j=0}^{r} c_{j} y^{(j)}=0
\end{array}\right.
$$

Remark 2 Taking $v$ as in (29) is useful to push down the time derivative of descent function (28) so that it becomes less than zero for all $x, u$ and $y, \dot{y}, \cdots, y^{(r)}$ except for $x=x^{s}$ where $\frac{\partial \beta^{r-1}\left(x^{s}\right)}{\partial x} g\left(x^{s}\right)=0$.

Theorem 1 Assume that system (1)-(2) has relative degree $r$ and satisfies Assumptions 1. Choose constants $c_{i}$ such that the polynomial

$$
p(s)=c_{o}+c_{1} s+\cdots+c_{r-1} s^{r-1}+c_{r} s^{r}
$$

is Hurwitz. Then, by using the dynamic control law 


$$
\dot{u}=-2 c_{r}\left(\sum_{j=0}^{r} c_{j} y^{(j)}\right) \frac{\partial \beta^{r-1}(x)}{\partial x} g(x)+v,
$$

with $v$ as in (32), $y$ and $x$ tend to zero if time $t$ goes to infinity.

Proof Consider equation (31) and what happens if $\sum_{j=0}^{r} c_{j} y^{(j)}$ is zero . Let $\sum_{j=0}^{r} c_{j} y^{(j)}=0, \forall t \geq t_{1}>0$. From Eq. (31) or (28), $\dot{F}\left(y, \dot{y}, \cdots, y^{(r)}\right)$ becomes zero. From equation (13) (the definition of descent function $F$ ), $\dot{F}\left(y, \dot{y}, \cdots, y^{(r)}\right)$ becomes zero. It means that our goal to minimize $\dot{F}\left(y, \dot{y}, \cdots, y^{(r)}\right)$ is achieved. Thus, if we choose $c_{j}, j=0, \cdots, r$ such that polynomial (12) is Hurwitz then $y$ goes to zero as $t \rightarrow \infty$.

By Assumption 1), if the modified steepest descent control is applied, the output of the system goes to zero and state $x$ becomes bounded.

\section{Example}

Consider the nonlinear system equation (SISO):

$$
\begin{aligned}
& \dot{x}_{1}=-x_{1}+x_{2}^{2} \\
& \dot{x}_{2}=-x_{2}+u \\
& y=x_{1} .
\end{aligned}
$$

For this system we have $\ddot{y}=x_{1}-3 x_{2}^{2}+2 x_{2} u$ and relative degree of the system is 2 at any point $x^{0} \neq 0$ ( relative degree of system is not well defined). System (35) is minimum phase [10].

Define descent function

$$
F_{0}(\ddot{y}, \dot{y}, y)=\left(c_{2} \ddot{y}+c_{1} \dot{y}+c_{0} y\right)^{2} .
$$

Then according to (4), the static control law is

$$
u=-\frac{1}{2 x_{2}}\left(x_{1}-3 x_{2}^{2}+\frac{1}{c_{2}}\left(c_{0} y+c_{1} \dot{y}\right)\right)
$$

and according to (34) and (29), the modified steepest descent control is

$$
\dot{u}=-4 x_{2} c_{2}\left(c_{0} y+c_{1} \dot{y}+c_{2} \ddot{y}\right)+v,
$$


where

$$
v=\left\{\begin{aligned}
\left\{\begin{array}{l}
-\frac{1}{2 c_{2} x_{2}}\left(c_{0} \dot{y}+c_{1} \ddot{y}+\right. \\
c_{2}\left(-x_{1}+x_{2}^{2}-\right.
\end{array}\right. & x_{2} \neq 0 \text { or } \sum_{j=0}^{2} c_{j} y^{(j)} \neq 0 \\
\left.\left.\left(-x_{2}+u\right)\left(6 x_{2}+2\right)\right)\right) & \\
0, & x_{2} \neq 0 \text { or } \sum_{j=0}^{2} c_{j} y^{(j)}=0
\end{aligned}\right.
$$

Consider the static control law (37). This static control law is fail if $x_{2}$ becomes zero. Simulation results are shown in Figure 1 for constants: $c_{0}=6$; $c_{1}=5 ; c_{2}=1$ and in Figure 3 for constants: $c_{0}=30 ; c_{1}=11 ; c_{2}=1$. In Figure 1, after time about $t=0,335$ and in Figure 3, after time about $t=0,0512$ computation stopped because the value of static control law (37) becomes infinity. Simulation results for the modified steepest descent control are shown in Figure 2 and in Figure 4. Thus, from simulation results, the modified steepest descent control success to handle the problems that arise if the relative degree of the nonlinear system not well defined.

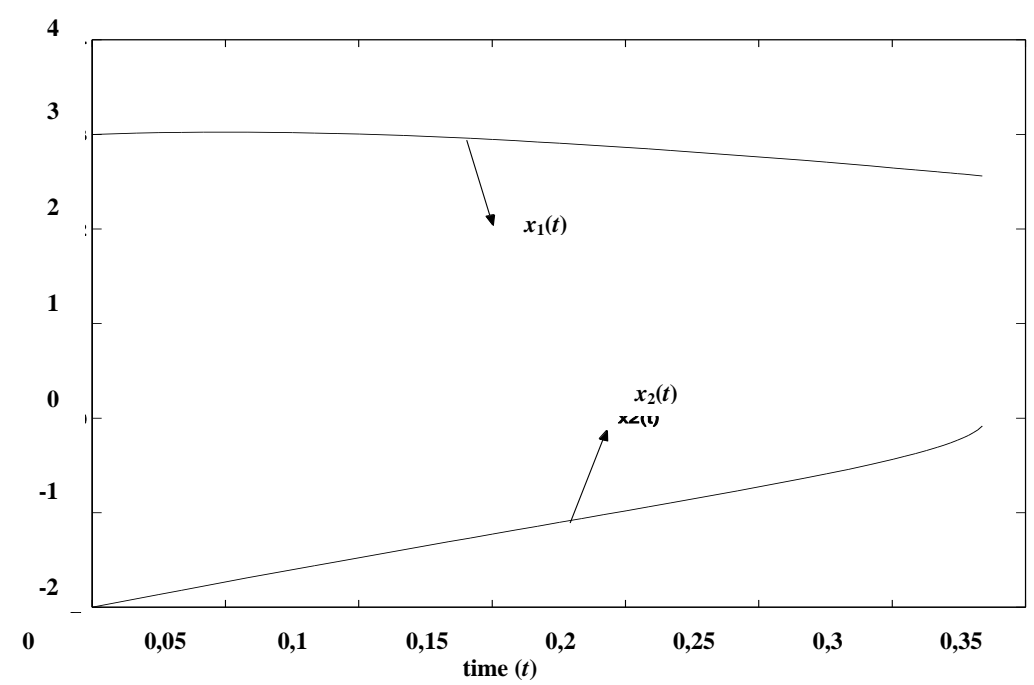

Figure 1 Static Control Law. Initial value: $x_{1}(0)=3 ; x_{2}(0)=-2$; Constants: $c_{0}=6 ; c_{1}=5 ; c_{2}=1$. 


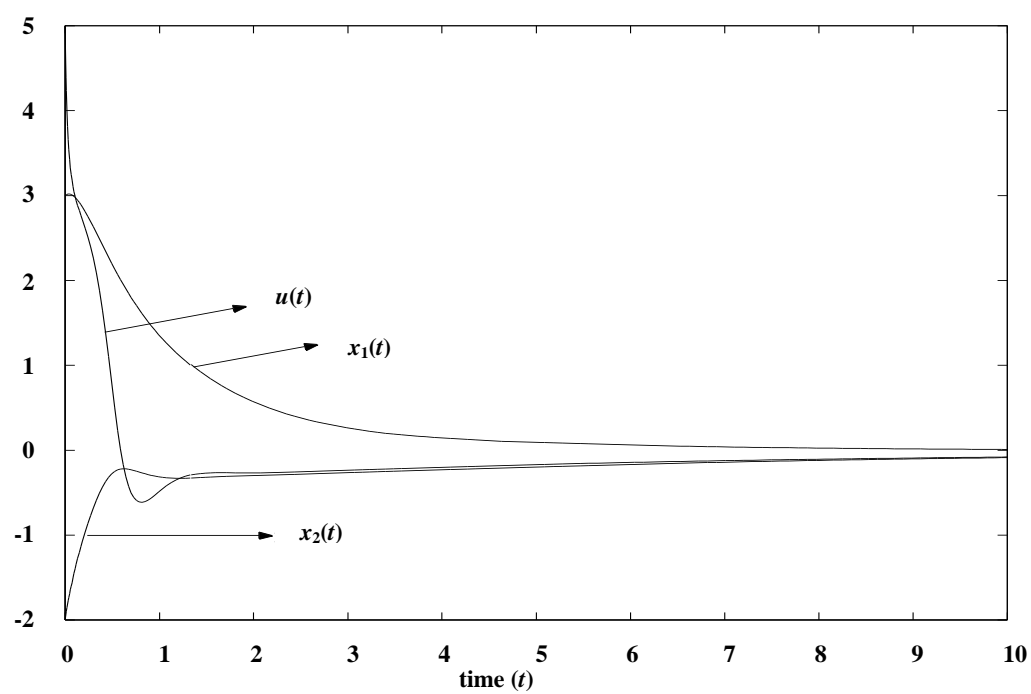

Figure 2 Modified Steepest Descent Control. Initial value: $x_{1}(0)=3 ; x_{2}(0)=-2 ; u(0)=5$; Constants: $c_{0}=6 ; c_{1}=5 ; c_{2}=1$.

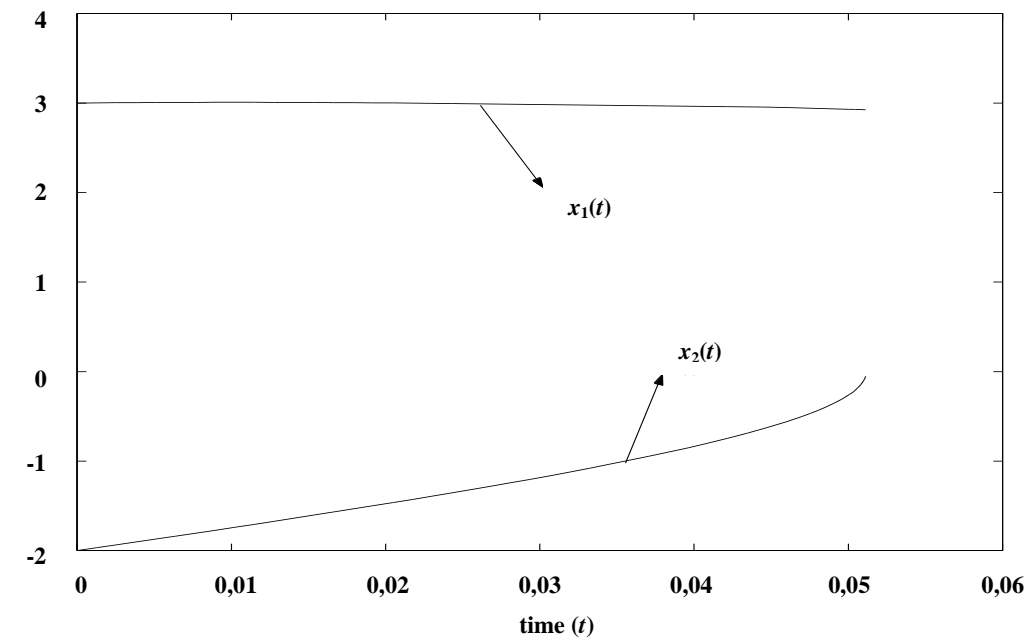

Figure 3 Static Control Law. Initial value: $x_{1}(0)=3 ; x_{2}(0)=-2$; Constants: $c_{0}=30 ; c_{1}=11 ; c_{2}=1$. 


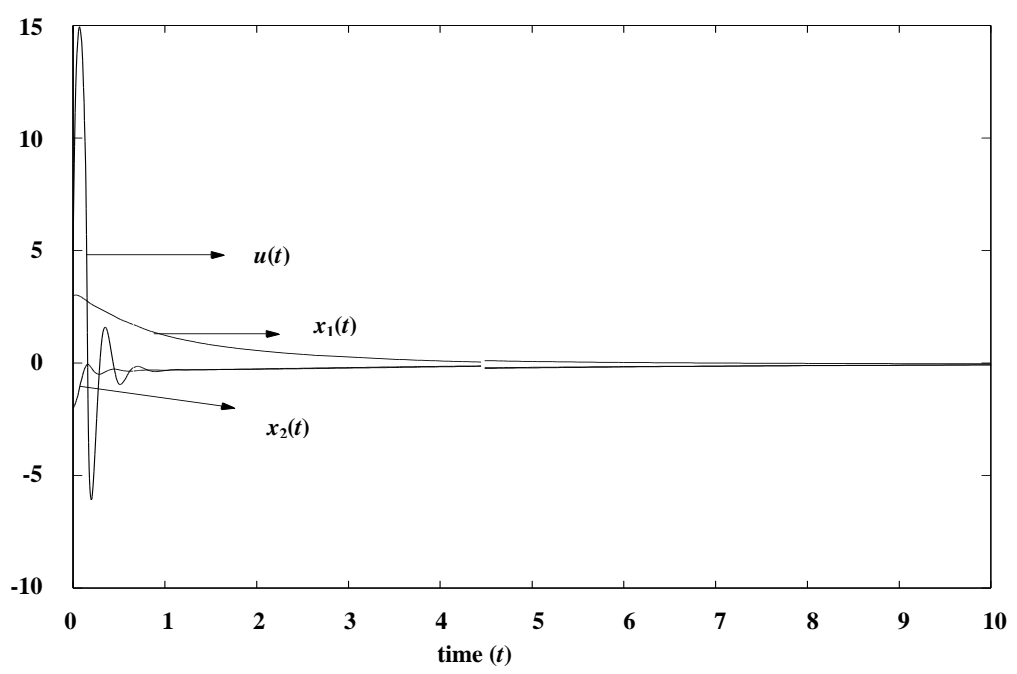

Figure 4 Modified Steepest Descent Control. Initial value: $x_{1}(0)=3 ; x_{2}(0)=-2 ; u(0)=5 ;$ Constants : $c_{0}=30 ; c_{1}=11 ; c_{2}=1$.

\section{Conclusions}

We have applied the trajectory following method for regulation output of affine nonlinear control system. By using this method we generate the modified steepest descent control which regulate the output of the system globally asymptotically. The modified steepest descent control is a dynamic feedback control and does not need relative degree of the system well defined. This is a superiority of the modified steepest descent control if we compare with the static control law which is obtained by input-output linearization technique.

\section{References}

[1] Tsinias, J. \& Kalouptsidis, N., Output Feedback Stabilization, IEEE Trans. Automat. Control, 35, 951-954, 1990.

[2] Artstein, Z., Stabilization with Relaxed Controls, Nonlinear Anal., 7, 1163-1173, 1983.

[3] Sontag, E.D., A "Universal" Construction of Artstein's Theorem on Nonlinear Stabilization, Syst. Contr. Lett.,13(2), 117-123, 1989.

[4] Pomet, J.B., Hirschorn, R.M. \& Cebuhar, W.A., Dynamic Output Feedback Regulation for a Class of Nonlinear Systems, Math. Control Signals Systems, 6, 106-124, 1993.

[5] Vincent, T.L. \& Grantham, W.J., Nonlinear and Optimal Control Systems, John Wiley \& Sons, Inc., New York, 1997. 
[6] Grantham, W.J. \& Chingcuanco, A.O., Lyapunov Steepest Descent Control of Constrained Linear Systems, IEEE Trans. Automat. Control, AC-29(8), 740-743, 1994.

[7] Naiborhu, J. \& Shimizu, K., Direct Gradient Descent Control for Global Stabilization of General Nonlinear Control Systems, IEICE Trans. Fundamental, E83-A(3), 516-523, 2000.

[8] Shimizu, K., Otsuka, K. \& Naiborhu, J., Improved Direct Gradient Descent Control of General Nonlinear Systems, Proc. of European Control Conference, 1999.

[9] Shimizu, K., Nukumi, H. \& Ito, S., Tracking Control of General Nonlinear Systems by Direct Gradient Descent Method, Preprints of the 4th Nonlinear Control Systems Design Symposium 1998, Enschede, The Netherlands, pp. 185-190, 1-3 July 1998.

[10] Isidori, A., Nonlinear Control Systems: An Introduction, Third Edition, Springer-Verlag Berlin, Heidelberg 1995.

[11] Khalil, H.K., Nonlinear Systems, Third Edition, Prentice Hall, New Jersey, 2002.

[12] Hirschorn, R.M. \& Davis, J.H., Output Tracking for Nonlinear Systems with Singular Points, SIAM J. Control and Optimization, 25(3), 547-557, 1988. 